\title{
Aging memories: Differential decay of episodic memory components
}

\author{
Lucia M. Talamini ${ }^{1}$ and Eva Gorree \\ Brain and Cognition Group, Department of Psychology, University of Amsterdam, Amsterdam 1018WB, The Netherlands
}

\begin{abstract}
Some memories about events can persist for decades, even a lifetime. However, recent memories incorporate rich sensory information, including knowledge on the spatial and temporal ordering of event features, while old memories typically lack this "filmic" quality. We suggest that this apparent change in the nature of memories may reflect a preferential loss of hippocampus-dependent, configurational information over more cortically based memory components, including memory for individual objects. The current study systematically tests this hypothesis, using a new paradigm that allows the contemporaneous assessment of memory for objects, object pairings, and object-position conjunctions. Retention of each memory component was tested, at multiple intervals, up to 3 mo following encoding. The three memory subtasks adopted the same retrieval paradigm and were matched for initial difficulty. Results show differential decay of the tested episodic memory components, whereby memory for configurational aspects of a scene (objects' co-occurrence and object position) decays faster than memory for featured objects. Interestingly, memory requiring a visually detailed object representation decays at a similar rate as global object recognition, arguing against interpretations based on task difficulty and against the notion that (visual) detail is forgotten preferentially. These findings show that memories undergo qualitative changes as they age. More specifically, event memories become less configurational over time, preferentially losing some of the higher order associations that are dependent on the hippocampus for initial fast encoding. Implications for theories of long-term memory are discussed.
\end{abstract}

[Supplemental material is available for this article.]

Autobiographical memories can persist over long periods of time, even a lifetime. However, recent event memories tend to be information dense and vivid, rendering a "filmic" quality that appears to be lacking in older memories. This change in memories over time may simply reflect an ongoing random loss of information, whereby the persistence of a memory fragment is mostly a function of encoding strength. On the other hand, certain elements of event memories may systematically persist longer than others, consequent, among other factors, to differential decay in the neural systems that encode them. If so, this would lead to qualitative differences in the contents of recent and remote memory.

While different aspects of events, for instance, objects, spatial metrics, smells, etc., are processed in widespread regions of the brain, their co-occurrence and temporal ordering is encoded by the hippocampal formation (McClelland et al. 1995; Eichenbaum 2000; Lisman et al. 2005; Talamini et al. 2005; Bachevalier and Nemanic 2008). However, neural network models predict that the hippocampus's high plasticity and nontopological wiring that enable fast storage of conjunctions also lead to fast overwriting of memory traces and, thus, a high decay rate of hippocampal representations (McClelland et al. 1995). Hence, the role of the hippocampus in memory is thought to be time-limited, and more enduring memory for associative aspects of events is thought to rely on the establishment of representations in other parts of the brain, in which memories decline at a slower rate (Alvarez and Squire 1994; Meeter et al. 2004; Meeter and Murre 2005; Murre et al. 2006). The occurrence of this process, termed systemlevel consolidation, is now supported by a considerable body of experimental evidence (Kim and Fanselow 1992; Moscovitch

\footnotetext{
${ }^{1}$ Corresponding author.
}

E-mail L.M.Talamini@uva.nl.

Article is online at http://www.learnmem.org/cgi/doi/10.1101/lm.024281.111. and Nadel 1998; Meeter and Murre 2004; Takashima et al. 2009; Winocur et al. 2010).

We propose that a change in the nature of memories over time may be related to a substantial loss of hippocampus-dependent associative information in this system-level consolidation step. After all, the hippocampal formation stores massive amounts of information each day, while the capacity of the nervous system for long-term memory storage is limited. Moreover, system-level consolidation is thought to be a competitive process wherein only the strongest hippocampal representations are likely to be reactivated and recoded, at the expense of weaker ones. Thus, it appears plausible that many, if not most, incidental associations featured in events will not undergo system-level consolidation but will be lost with degradation of the hippocampal representation. If the hippocampal decay rate exceeds the rate in brain areas coding for the separate components of events, this would lead to a preferential loss of configurational information from event memories, including precise knowledge of the co-occurrence of objects, their spatial location, and temporal order information (for related viewpoints, see Meeter and Murre 2004; Riccio and Joynes 2007; Winocur et al. 2010).

In support of this notion, several studies have observed memory changes over time that could reflect a preferential loss of hippocampus-dependent information. For instance, recent autobiographical memories receive higher "remember" ratings on the remember-know scale than old ones (Catal and Fitzgerald 2004; Tunney 2010). This might reflect lesser availability of hippocampus-encoded spatial and temporal context for older events and, thus, a higher reliance on familiarity in recognition.

Also, experiments in rodents have shown that partial changes to the experimental apparatus or task environment have progressively lesser effects on retrieval of conditioned responses at longer delays from encoding (Perkins and Weyant 1958; 
McAllister and McAllister 1963; Zhou and Riccio 1996; Anderson and Riccio 2005; Wiltgen and Silva 2007; Winocur et al. 2009; Wiltgen et al. 2010). This suggests a gradual loss of memory for some stimulus attributes guiding a learned target response, with preferential maintenance of the target response itself. Importantly, two such studies showed that a reduction of context effects on retrieval over time coincided with reduced involvement of the hippocampus in retrieval (Winocur et al. 2009; Wiltgen et al. 2010). This is in line with loss of hippocampus-dependent configurational information with system-level consolidation.

Finally, a few experiments have assessed differential forgetting of "what," "when," and "where" aspects of autobiographical memories, finding stronger persistence of "what" information with respect to the spatiotemporal context reflected in the "where" and "when" questions. Again, the preferential loss of when and where information could be taken to reflect loss of hippocampus-dependent memory components.

However, none of the aforementioned studies directly compares forgetting of single item and configural memory elements, and, in fact, findings thus far could also be explained by memory mechanisms in which the relative endurance of specific memory components is primarily due to differences in encoding strength. In the case of temporal gradients in remember-know ratings, remember responses may simply require a more intact memory representation that familiarity responses (Wais et al. 2008). In the conditioning studies, an assembly of associative data may initially support response retrieval. As more weakly encoded links are lost, remaining, more strongly encoded ones may still suffice to support the conditioned response. Finally, the autobiographical memory studies are confounded with both initial differences in encoding strength and considerable repetition effects, while, in addition, the mapping of tested memory components onto specific brain circuits is not straightforward. Thus, the occurrence and nature of any systematic changes in memory over time remain speculative.

Here, we aim to assess how memory content might change over time by directly comparing forgetting of different memory elements after initial encoding. We hypothesize that forgetting is not evenly distributed over different memory components (random decay) but occurs preferentially for configurational information, with relative sparing of nonconfigurational components. Configurational information here refers to the spatial and temporal relations between event components that are themselves encoded in higher order cortical areas projecting to the hippocampus (e.g., objects, persons, egocentric spatial features, smells, etc.).

To investigate our hypothesis, we developed a paradigm that simultaneously tests memory for individual objects and configu- rational aspects of simple scenes. During encoding, subjects learn a set of object-to-position and object-to-object associations. At five different intervals following training, from $5 \mathrm{~min}$ up to 3 mo, they are tested for recognition of different aspects of the learned information, including objects (against foils), object detail (against similar new objects), object-position pairings (against repositioned objects) and object-object pairings (against recombined pairs). All tasks, except detailed object recognition, are matched for task difficulty to preclude alternative interpretations of findings in terms of differences in initial encoding strength.

Notably, the object pairs in the task are not semantically associated, nor are there meaningful relations between objects and their position. This fact, and the testing of memory for these conjunctions through associative recognition, precludes familiaritybased contributions to retrieval (Zhu et al. 1995; Aggleton et al. 2005; Vann et al. 2009). Thus, the retrieval of object-position and object-object conjunctions should rely entirely on the formation of new associative links by the hippocampus. On the other hand, object recognition can be performed using both recollection, which is thought to require the hippocampus, and familiarity, which relies importantly on parahippocampal and other mediotemporal cortices. Familiarity should support retrieval even when hippocampal links are lost (Yonelinas 2002).

Memory for object detail was assessed to test the alternative hypothesis that perceptual details are forgotten faster than more global aspects of an event. According to our view, however, global and detailed object memories rely on similar neural mechanisms that do not strictly require the hippocampus and thus would decline at a similar rate. On the other hand, both types of tested associations are thought to depend crucially on hippocampal encoding and are thus expected to decline at a faster rate than both types of object memory.

\section{Results}

The discrimination between learned items and foils in recognition, reflected in $\mathrm{d}^{\prime}$, is considered an accurate measure of recognition performance. Thus, $\mathrm{d}^{\prime}$ is used as the primary measure to test our hypotheses. $\mathrm{H}, \mathrm{FA}$, and $\mathrm{C}$ are used to evaluate memory performance in more detail.

The results of full factorial (retention interval by memory component) repeated measures ANOVAs on the recognition data are given in Table 1. In the following, however, we will focus mainly on the outcome of the analyses with respect to the differential decay of memory components, as reflected in the interaction effect of the factors Time and Memory component. Unless otherwise specified, all mentioned contrast analyses regard this

Table 1. Test results from the two-factor repeated measures ANOVA

\begin{tabular}{|c|c|c|c|c|c|c|c|c|c|c|c|c|c|c|c|c|}
\hline & \multicolumn{4}{|c|}{$d^{\prime}$} & \multicolumn{4}{|c|}{ Hit rate } & \multicolumn{4}{|c|}{ False alarm rate } & \multicolumn{4}{|c|}{ C } \\
\hline & Signif. & $F$ & df & $\mathbf{d f}_{\text {error }}$ & Signif. & $F$ & df & $\mathbf{d f}_{\text {error }}$ & Signif. & $F$ & df & $\mathbf{d f}_{\text {error }}$ & Signif. & $F$ & df & $\mathbf{d f}_{\text {error }}$ \\
\hline \multicolumn{17}{|l|}{ Within-subject effects } \\
\hline Time & *** & 242.0 & 4.0 & 216.0 & $* * *$ & 277.4 & 4.0 & 216.0 & $* * *$ & 20.3 & 4.0 & 216.0 & $* * *$ & 102.2 & 3.3 & 177.9 \\
\hline Memory & $* * *$ & 35.2 & 2.5 & 133.4 & $* * *$ & 4.0 & 1.6 & 88.4 & $* * *$ & 46.6 & 2.5 & 133.2 & $* * *$ & 35.5 & 2.4 & 130.7 \\
\hline Time $\times$ memory & *** & 5.7 & 6.9 & 370.7 & $* * *$ & 7.8 & 4.9 & 262.1 & $* * *$ & 3.7 & 7.8 & 418.6 & $* * *$ & 6.0 & 7.2 & 388.1 \\
\hline \multicolumn{17}{|c|}{ Within-subject contrasts (time contrast $\times$ memory contrast) } \\
\hline General obj. $\times$ detailed obj. & $*$ & 5.7 & 1 & 54 & - & - & - & - & ns & 3.8 & 1 & 54 & * & 5.7 & 1 & 54 \\
\hline General obj. $\times$ obj.-location & $* * *$ & 11.7 & 1 & 54 & ** & 7.3 & 1 & 54 & * & 6.6 & 1 & 54 & ns & 0.0 & 1 & 54 \\
\hline General obj. $\times$ obj.-obj. & *** & 18.1 & 1 & 54 & *** & 86.9 & 1 & 54 & ns & 0.9 & 1 & 54 & $* * *$ & 50.3 & 1 & 54 \\
\hline Obj.-location $\times$ obj.-obj. & ns & 0.1 & 1 & 54 & ** & 9.3 & 1 & 54 & $* * *$ & 12.7 & 1 & 54 & $* * *$ & 22.9 & 1 & 54 \\
\hline Detailed obj. $\times$ obj.-location & *** & 25.1 & 1 & 54 & - & - & - & - & $* * *$ & 18.0 & 1 & 54 & ns & 2.2 & 1 & 54 \\
\hline Detailed obj. $\times$ obj.-obj. & $* * *$ & 39.1 & 1 & 54 & - & - & - & - & ns & 1.5 & 1 & 54 & *** & 26.8 & 1 & 54 \\
\hline
\end{tabular}

Significance: $\left({ }^{* *}\right) P \leq 0.001 ;(* *) P \leq 0.01 ;(*) P \leq 0.05 ;$ (ns) $P \geq 0.05$. 
Table 2. $d^{\prime}$ for different memory components in the first retrieval session

\begin{tabular}{lcc}
\hline Memory component & Mean & St. dev. \\
\hline Object (general) & 3.12 & 0.44 \\
Object (detail) & 2.13 & 0.95 \\
Object-location & 2.94 & 0.58 \\
Object-object & 2.91 & 0.65 \\
\hline
\end{tabular}

interaction. In addition, some statistics concerning task difficulty are presented hereafter.

\section{Task difficulty}

The different recognition tasks in the paradigm were matched for initial recognition performance (Table 2), based on pilot studies, to rule out interpretations of results in terms of task difficulty. An exception concerns detailed object recognition, in view of practical limitations related to the overall design of the study. The $\mathrm{d}^{\prime}$ data of the first retrieval session (5-min retention interval) were analyzed through repeated-measures ANOVA, with the four memory components as a within-subjects factor (main effect of Memory component: $\left.F_{(2.55,137.55)}=33.10, P<0.001\right)$ and contrast analyses between the memory components. As expected, performance on detailed object recognition is significantly lower than on every other recognition task ( $\left.P^{\prime} \mathrm{s}<0.001\right)$. In addition, there is slightly higher performance on general object recognition compared to the two associative tasks ( $P^{\prime} \mathrm{s}<0.05$ ), while the difference between the associative tasks is not significant.

\section{$\mathrm{d}^{\prime}$ scores}

The evolution of $\mathrm{d}^{\prime}$ scores over time (Fig. 1) shows substantial differences between memory components (Time $\times$ Memory Component: $\left.F_{(6.9,370.7)}=5.7, P<0.001\right)$, with stronger overall decay of configurational than nonconfigurational information. ${ }^{2}$ Indeed, contrasts between the different levels of Memory component (Table 1) indicate significant differences in decay between general object recognition and both configurational memory components $\left(P^{\prime} \mathrm{s}<0.001\right)$ and also between detailed object recognition and both configurational memory components ( $\left.P^{\prime} \mathrm{s}<0.001\right)$, whereas the contrast between the two configurational memory components is not significant.

Moreover, the development of decay over time appears to differ between configurational and nonconfigurational memory components: the decay curves for object memory level off around the 1-mo time point, whereas the configurational memory components continue decaying up until the final measuring point at 3 mo after training. Thus, differential decay is primarily apparent during the later part of the retention interval. This is confirmed by stepwise contrasts on the time factor, in which each level of the time factor (each retrieval session) is compared to the previous one: decay of general object recognition and the two configurational memory components only differs significantly between the 1 -mo and 3-mo time points $\left(P^{\prime} \mathrm{s}<0.01\right)$. Similarly, decay of detailed object memory differs significantly from object-position memory between the 1 -mo and 3 -mo time points $(P=0.003)$

\footnotetext{
${ }^{2}$ The calculation of $d^{\prime}$ involves replacement of hit rates of 1 with lower scores (see Materials and Methods section). This leads to some underestimation of actual performance on the shortest retention rates, which contain a relatively high proportion of perfect performance scores (in view of the long-term character of the study, this was very hard to avoid). This, in turn, leads to a slight $S$-shape in the early part of some of the $d^{\prime}$ retention curves, which in all likelihood represents an artefact of the correction procedure.
}

and from object pair recognition between the 1 -wk $(P=0.04)$ and 3-mo time points $(P=0.007)$.

It might be noted that there is also a slight difference in overall decay between general and detailed memory, with somewhat lesser decay of the latter (contrast between the two memory components: $P=0.02$ ). However, there are no significant differences in the shape of the two decay curves (stepwise contrasts on the time factor are all nonsignificant [n.s.]). This difference in overall decay might be related to the relatively low immediate recognition scores for detailed object memory, reflecting a methodological artefact rather than true differences in decay mechanisms for the two memory components.

\section{Hit rates}

Figure 2 shows the decay of $\mathrm{H}$ over time for the different memory components. The trained items for general and detailed object recognition are the same, so there is only one $\mathrm{H}$ for object recognition. Again, there is a significant interaction effect between the Time and Memory components $\left(F_{(12,648)}=8.49, P<0.001\right)$. Results are similar to the $\mathrm{d}^{\prime}$ findings in that decay of $\mathrm{H}$ is much stronger for the configurational memory components than for object recognition (contrasts: object vs. object-position, $P=0.009$; object vs. object-object, $P<0.001$ ) and that the difference in decay manifests itself mostly during the later part of the retention period. Indeed, considering contrasts between adjacent levels of the time factor, object memory differs significantly from objectposition memory after the 1 -mo time point $(P<0.01)$, and from object-object memory after the 1 -wk time point ( $1 \mathrm{wk}$ to $1 \mathrm{mo}$, $P<0.05 ; 1$ mo to 3 mo, $P<0.001$ ).

In addition to the large difference between configurational and nonconfigurational memory components, there is also a smaller difference in decay between the two configurational memory components. Indeed, $\mathrm{H}$ decays somewhat more strongly for object-object associations than for object-position associations (contrast: $P=0.004$ ). Considering the contrasts between different time steps, differential decay is significant between the 1 -wk and 1 -mo time points $(P<0.01)$. As we shall see further on, this is related to a difference in response bias between the two associative memory components.

\section{False alarm rates}

Figure 3 shows the development of FA over time for the different memory components. As expected, most false alarms are made in response to the lures, used to assess detailed object recognition. However, while FA for lures starts out high during the first retrieval session, it does not increase much further during the remainder of the sessions. In fact, most memory components show a relatively

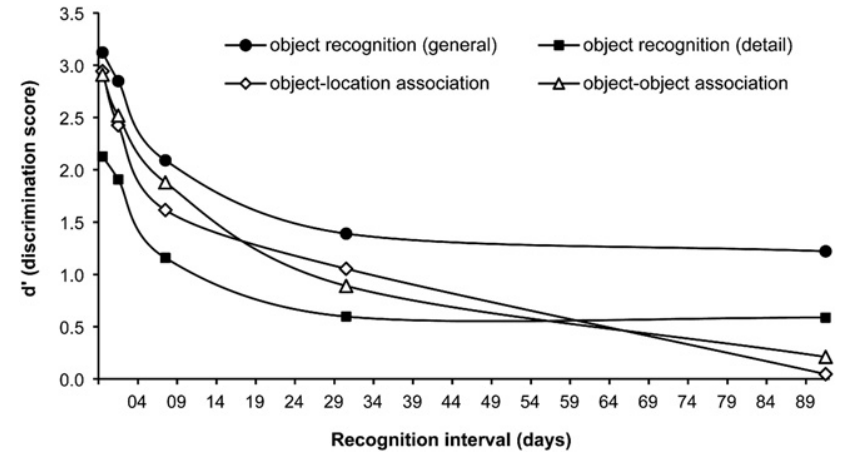

Figure 1. Evolution of $d^{\prime}$ scores over time for general object memory, detailed object memory, object-object pairs, and object-location pairs. 


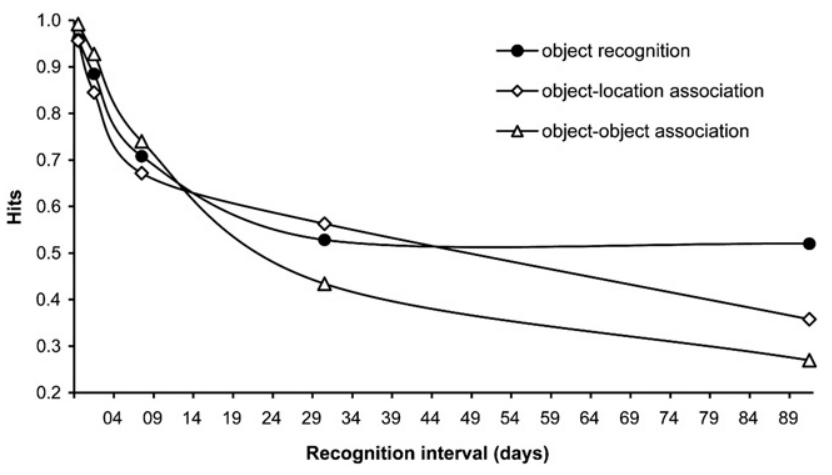

Figure 2. Evolution of hits over time for object memory, object-object pairs, and object-location pairs.

mild increase of FAs over time. A steeper increase is observed only for object-position associations. This is reflected in a significant interaction effect between Time and Memory components $\left(F_{(7.75,418.60)}=3.74, P<0.001\right)$, with significant contrasts between object-position recognition and all other memory components (vs. general object memory, $P=0.013$; vs. detailed object memory, $P<0.001$; vs. object-object memory, $P<0.001$; remaining contrasts n.s.).

It thus appears that changes in FA over time contributed only modestly to deteriorating recognition performance and do not underlie the differences between configurational and nonconfigurational memory decline. However, the combined $\mathrm{H}$ and FA data do point out a difference between the two associative memory components featured in the task: during late retention, both $\mathrm{H}$ and FA in the object-position task are relatively higher than in the object-to-object task. Thus, while discrimination is similar for these two memory components, response bias at longer delays seems to be more liberal for the position task. This point will be addressed more formally in the next section.

It should be noted that the sharp fluctuation in the initial part of the curve for detailed object recognition is probably related to the study's methodology: subjects became aware of the possibility of lures during the first session. This may have led to a more conservative response strategy and concomitant decrease of FAs in the second session. The reason for a similar fluctuation (but in the opposite direction) in the early part of the objectobject curve is currently unclear.

\section{Response bias}

To address response bias formally, $\mathrm{C}$ was calculated. As shown in Figure 4, response bias became more conservative with time for all memory components, especially during the early part of the retention period. In fact, as of the 1-wk time point, all memory components are, on average, at or above zero (i.e., neutral to conservative response bias).

However, some differences between memory components may be noted. As expected, the most liberal response bias values throughout the experiment are found for detailed object memory (main effect of Memory component: $F_{(2.4,130.7)}=35.5, P<0.001$; contrasts for detailed object memory vs. all other memory components: $P$ 's $<0.001)$. More importantly, the analysis confirms a significant difference in the development of response bias between the two configurational memory components (Time $\times$ Memory component: $F_{(7.2,388.1)}=6.0, P<0.001$; contrast object-object vs. object-position, $P<0.001$ ): the increase is significantly larger and reaches higher (more conservative) end values for object-object memory; responses for object-position conjunctions remain relatively liberal, even though they start out more conservative than responses to object pairs. Apparently, the recognition of an object leads to relatively liberal responses with regard to memory for its location.

\section{Memory fragmentation over time}

According to our differential decay analyses, the configural links between event components are lost progressively and preferentially. Episodic memories thus become decontextualized or "fragmented" over time. Our setup allows quantification of the amount of fragmentation over time for object-position conjunctions, because, in the object position task, recognition of both object and object position were tested for each item.

The percentage of recognized objects that was remembered with the appropriate position was calculated for each retention interval (\# of correctly recognized positions/\# of correctly recognized objects $\times 100$ ). In this analysis, we only considered responses to objects placed in their original position at test. As expected, the percentage of recognized objects for which the position was also recognized decreased progressively over time, from $96 \%$ right after training to $36 \%$ after 3 mo (Fig. 5). Interestingly, after the first week from encoding, fragmentation appears to progress linearly.

\section{Discussion}

We have assessed memory retention for different components of scenes over a 3-mo period. In line with our hypothesis, we find stronger forgetting of configurational components with respect to featured objects. The difference in forgetting rate is not immediately apparent but develops between $1 \mathrm{wk}$ and 1 mo after encoding, at which point the retention curves for configurational and nonconfigurational components take on significantly different trajectories.

Differences in task difficulty or initial encoding strength do not present a likely explanation for these findings, as initial recognition scores are similar for general object memory and the configurational memory tasks. Furthermore, detailed object memory is better retained over the 3-mo interval than either of the configurational memory components, despite lower initial recognition rates. Thus, our findings strongly suggest faster decline of hippocampus-dependent, configurational memory elements than of nonconfigurational memory elements, retrieval of which should also be supported by extrahippocampal cortices, in the absence of an intact hippocampal trace.

The results of this study have implications for classical consolidation theory, which holds that declarative memory traces become increasingly resistant to decay as they age. This notion stems from two types of observations. First, forgetting functions are typically characterized by an ever decreasing proportional rate of

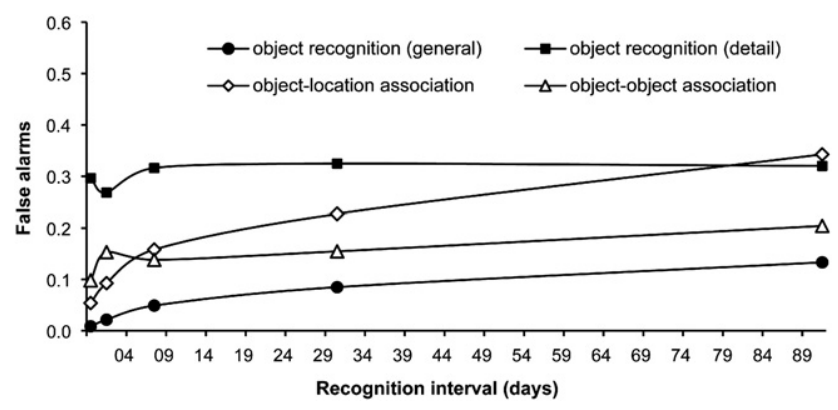

Figure 3. Evolution of false alarms over time for object memory, detailed object memory, object-object pairs, and object-location pairs. 


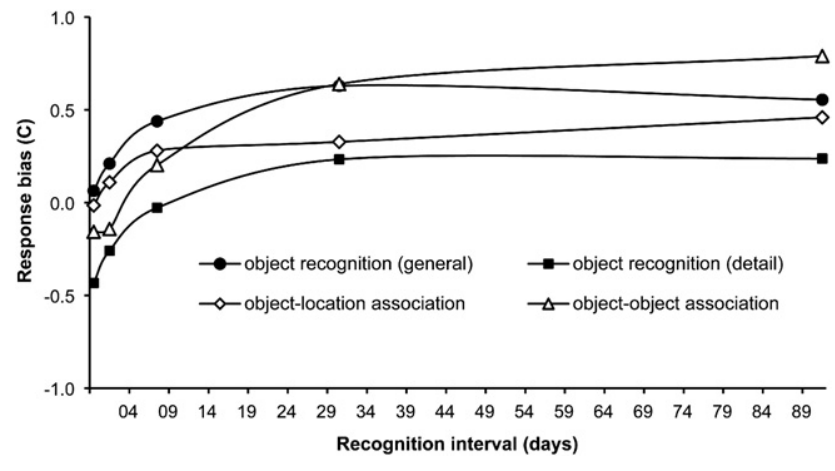

Figure 4. Evolution of $\mathrm{C}$ over time for object memory, detailed object memory, object-object pairs, and object-location pairs.

decay (Ebbinghaus 1885; Wixted 2004b). Second, following brain damage, especially hippocampal damage (Scoville and Milner 1957; Squire et al. 2001; Manns et al. 2003), temporally graded retrograde amnesia may occur, with greater loss of recent memories than older ones (Ribot 1881; Squire et al. 1975; Brown 2002). Both findings might be expected if memories consolidate with longer survival (for a detailed discussion, see Wixted 2004a).

A closer look at the retention curves in our experiment reveals that, for object memory, proportional forgetting appears to decrease progressively with time, in line with previous findings. On the other hand, retention of configurational memory components starts off curvilinear but progresses toward a more linear trajectory between $1 \mathrm{wk}$ and 1 mo after encoding. Also, our memory fragmentation analysis, which directly reflects the configurational intactness of memories over time, shows entirely linear decay in later parts of the retention period. This means that configurational memory retention conforms neither to power functionlike forgetting ${ }^{3}$ nor to classical notions of long-term consolidation. Indeed, the linear forgetting in the late part of the retention curve is difficult to reconcile with any type of overall memory consolidation.

Linear forgetting has, to our knowledge, not been reported previously. The probable reason is that memory paradigms in earlier, long-term forgetting studies allowed for contributions of recollection, familiarity, and sometimes also semantic memory to retrieval. The combined contributions from linearly and curvili-

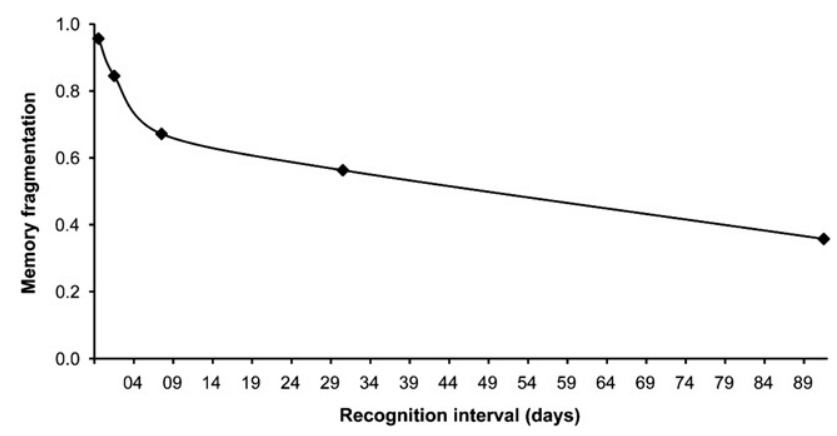

Figure 5. Memory fragmentation over time: the percentage of recognized objects that was remembered with the appropriate position for each retention interval.

${ }^{3}$ The Supplemental figure shows $\mathrm{d}^{\prime}$ plotted on a logarithmic-logarithmic scale.
The plot indicates that, on the whole, decay progresses faster than a power The plot ind
function. nearly declining memory components would have led to curvilinear forgetting. Differential forgetting of item and associative memory, using associative recognition to assess the latter, has been investigated previously but only for retention intervals up to $1 \mathrm{wk}$. These studies found faster decline of item recognition compared to associative recognition for retention intervals in the short-term memory domain (Hockley 1991, 1992) and, in line with our own findings, similar decline for retention intervals between 30 min and 1 wk (Hockley and Consoli 1999). Here, we show that, with even longer retention intervals, associative information deteriorates more rapidly than item information.

Our findings thus show that configurational memory components are initially subject to consolidation (proportional decay decreases over time in the early part of the curve ${ }^{4}$ ) but not beyond the first week or so after encoding. Can this be reconciled with current models of system-level consolidation? To answer this question, let us first consider that consolidation over days and weeks is likely to involve system-level processes. Indeed, fMRI experiments in humans suggest that some hippocampocortical recoding may occur as soon as $24 \mathrm{~h}$ after encoding (Takashima et al. 2006, 2009).

Having said this, our findings do not easily fit standard system-level consolidation theory, which entails a two-stage process by which newly acquired associations are, over time, recoded from a labile hippocampus-dependent to a more stable hippocampus-independent code. Our results may, however, be explained assuming a more graded consolidation process, involving multiple representational states of increasing stability. Such graded consolidation might be seen as progressing along a tree-like hierarchy of memory stores, with the hippocampus at the top and lower order cortices at the bottom-each store forming a potential consolidation hub for associations between event features held in lower order stores, and each store having its own parameters for decay and for induction of memory representations in subsequent stores. Within the body of relational data that constitutes the originally encoded episodic memory, different associations may follow different paths down this "consolidation tree," depending on their information content, and reach different degrees of consolidation depending on initial encoding strength and interactions with preexisting information (for relevant modeling accounts, see Murre et al. 2006; Battaglia and Pennartz 2011).

For incidental and relatively meaningless configurations, like those featured in our paradigm, the hippocampal representation may degrade relatively fast, and system-level consolidation may not be sustained beyond certain higher-order association areas, from which information may still be lost within days or weeks. On the other hand, consolidation dynamics may be more protracted, extending further down the tree, in the case of biologically relevant information (stronger encoding, synaptic consolidation, and rehearsal) or when the new material can be related to existing information. Formal modeling would have to be employed to assess whether a model of this type could, indeed, simulate our forgetting findings.

Interestingly, a few recent studies in rats (Peyrache et al. 2009; Tse et al. 2011) and unpublished observations of our own in humans (CCG Sweegers, A Takashima, G Fernández, and LM Talamini, in prep.) are in line with graded system-level consolidation, showing that, especially for information that reflects

${ }^{4}$ Extrapolation of decay rate over the first $24 \mathrm{~h}$, assuming a constant proportional decay rate, results in much lower $\mathrm{d}^{\prime}$ values after $1 \mathrm{wk}$ than the observed values. For object-location memory, decay rate over the first $24 \mathrm{~h}=24 \%$, extrapolated $d^{\prime}$ after $1 \mathrm{wk}=0.91$, and observed $\mathrm{d}^{\prime}$ after $1 \mathrm{wk}=3.2$. For object-to-object memory, decay rate over the first $24 \mathrm{~h}=20 \%$, extrapolated $\mathrm{d}^{\prime}$ after $1 \mathrm{wk}=1.35$, and observed $\mathrm{d}^{\prime}$ after $1 \mathrm{wk}=5.08$. 
regularities in the environment, involvement of certain meso- or neocortical association areas in retrieval may increase over time. This may reflect a shift of the associational hub for certain memory components from the hippocampus to other higher order associative brain areas as an early step in the consolidation process (Nieuwenhuis and Takashima 2011).

Our results also offer more practical insights into people's memory capacities and how these evolve over time. Interestingly, people appear to be about as proficient, shortly after exposure, at remembering objects and configurational aspects of scenes (notably, all memory subtasks were equivalent with respect to the number of items to be encoded and item presentation times). Only visual details of objects were then poorly remembered, perhaps as a consequence of lesser encoding. However, in the longer term (i.e., a few months after encoding), subjects are effectively left with some general object memory and very little else. This is due to the preferential long-term decline of configurational information and the low initial encoding of object detail so that, despite relatively good retention of object detail (similar to retention of general object information), very little such information is eventually available.

With respect to the reliability of long-term memory, it might first be noted that response bias becomes more conservative over time for all memory components. Thus, with time the number of false positive answers diminishes with respect to the number of correct answers. Even so, the absolute number of false alarms increases over time. For all practical purposes, this implies that the chance that a person erroneously recognizes an object, the co-occurrence of objects, or the location of an object increases over time.

False recognition of remote event information is especially likely in two situations: first, when an object conceptually resembles an encountered object (as might be expected); second, and perhaps more interesting, for the location of familiar objects. Apparently, recognition of an object installs a tendency to also recognize the presentation location as the original one. A more mechanistic account for this phenomenon is offered in a previous modeling study by our group (Talamini and Meeter 2009), which showed that, under certain circumstances, object cues outweigh visuospatial cues and dominate retrieval, leading to recognition of an episode despite nonmatching spatial context cues. ${ }^{5}$

Some limitations of the study should, at this point, be addressed. First, in view of the long-term character of the study, some ceiling and floor effects, at the shortest and longest retention intervals, respectively, could not be avoided. Second, subjects' repeated memory testing could represent a potential confound, in view of contextual cueing. However, we expect any effects to be slight, as the nature of the material should make it difficult to retrieve through free recall. Furthermore, subjects performed experimental sessions in self-chosen environments which could vary from session to session and were often familiar. These circumstances should reduce the efficacy of context cues as retrieval cues. A final factor that should be mentioned is the pairing of multiple objects to each screen location, which might have had some influence on object-location retention in view of cue overload. While slight influences of these factors on forgetting characteristics cannot be excluded, explanations of our main findings in terms of these potential confounds appear highly unlikely.

\footnotetext{
${ }^{5}$ This tendency originates from the differential wiring of object and spatial input to the hippocampus. It is especially apparent when connectivity in these pathways is reduced, as is the case in schizophrenia. A similar situation might arise in healthy subjects when hippocampal links, with time, start to fade and connectivity between object and visuospatial components of an event is reduced at a functional level.
}

In conclusion, we have shown that over time, our memory of events changes not only quantitatively, but also qualitatively, through preferential loss of configurational information. We have argued that this is likely related to high decay rates in the hippocampus, which encodes this type of information, and to limited access of hippocampal memory traces to system-level consolidation processes. This pattern in long-term forgetting appears to make sense from a functional perspective. Indeed, most of the new associations featured in events are incidental: they neither reflect general organization principles of the environment, nor predict biologically relevant stimuli. Such associations are only of interest in the short to intermediate term. For instance, to keep track of one's surroundings as long as one is navigating them, to remember multiple substeps toward goals, or to understand current discourse against earlier parts of the conversation. Only a very limited part of the new associations stored each day would have general relevance and merit preservation in the term of months and years.

Pertaining to theories of long-term memory, our findings emphasize the nonunitary nature of event memories and show that strictly relational memory components do not conform to power function-like forgetting and standard notions of consolidation. These findings urge us to consider more comprehensive models of consolidation than those proposed thus far. Such models would have to accommodate the differential decay of different memory components. They might entail graded consolidation along tree-like neural hierarchies and differential consolidation routes for different memory components.

\section{Materials and Methods}

\section{Participants}

Fifty-eight adults (age 18-65) with a medium to high educational level participated in this internet-based memory experiment. They were medication-free and had no (history of) neurological or psychiatric disorders. After registration on our web site for the first test session, subjects were informed via email when they had to log in on their individual test-account for a follow-up session.

Two people abandoned the experiment halfway for personal reasons. One person exceeded the given time intervals for all sessions. Analyses were conducted on the data of the 55 remaining subjects (23 males, 32 females; average age: 31.7 , st. dev.: 12.2). The local ethical committee approved of the experiment.

\section{Stimuli}

We used 200 color photographs of everyday objects, selected from a wide variety of categories. Differences in natural size of objects were somewhat decreased for the purpose of visibility. Fifty objects were presented, positioned in various locations on the floor of a virtual room. Apart from the test item, the room was empty and contained no distinctive features. The viewpoint was from one of the ends of the room, elevated from the floor by $\sim 60 \%$ of the room's height. The size of objects was adapted to the "depth" of their position in the room, to enhance the 3D illusion. Another one hundred objects were presented in semantically unassociated pairs (50 pairs), at fixed positions in the middle of the room. The remaining 50 objects served as foils in the object recognition task. Half of these strongly resembled one of the training objects and will henceforward be referred to as "lures." Lures differed from the respective list items in visual details, including small variations in shape, color, or patterning, not on functional aspects nor in design style.

\section{Memory task}

The experiment was performed over five sessions. The first session contained training on all test items and the first memory test at 
5 min following encoding. The subsequent four sessions tested memory at $24 \mathrm{~h}, 1 \mathrm{wk}, 1 \mathrm{mo}$, and 3 mo after training.

The memory task consisted of two subtasks: an object-to-position task and an object-to-object task. The first task measured three types of memory: general object recognition, detailed object recognition, and recognition of object-position conjunctions. The second measured recognition of object pairings. The order in which the subtasks were presented was randomized over subjects and over sessions. The order in which items appeared, both during training and during memory tests, was also randomized over subjects and sessions. During training on each subtask, every item was shown three times for 5 sec each time. Every trained item and foil was shown only once during the recognition tests.

\section{First session}

In the first session, subjects were presented with the two subtasks, separated by a 10 -min break, during which they were instructed not to discuss the test.

During training on the object-to-position task, 50 single objects were shown sequentially, each at one of nine possible positions in the virtual room. Training was followed by a simple math task (5 min), after which recognition was tested. To this purpose, 20 single-object items were presented. Ten of these were the same as those shown during training, but five were shown in the same position and the other five at another position. The remaining ten items were foils. Five of these were entirely new objects; the other five were lures, i.e., variants of learned objects (e.g., a modern blue desk lamp and a very similarly shaped silver-gray desk lamp). Following each item's presentation, subjects had to answer "Yes" or "No" to the following question(s): "Was this object shown in the training phase?" If they answered "Yes," the following question was presented: "Is the object in the same position as in the training phase?"

For the object-to-object task, subjects were presented with a sequence of 50 object pairs placed at fixed positions in the middle of the room. Following another math task ( $5 \mathrm{~min})$, recognition of object-object links was tested through presentation of 10 object pairs. Five of these were exactly the same as in the training phase, while five showed objects from the training phase that had been reorganized into new pairs. The objects always kept their original position, at the left or right of the room's center point. Following each item, subjects had to answer "Yes" or "No" to the following question: "Were these objects shown together in the training phase?"

\section{Sessions 2-5}

In each following session, subjects were again presented with the two recognition subtasks (object-to-position task and object-toobject task). As the recognition tasks generally took only a few minutes, there was no break between the two tasks.

\section{Data analysis}

Data were excluded from the analyses when subjects exceeded the time limits for session intervals, which were set at $25 \%$ deviation from the appointed time intervals. ${ }^{6}$ Thus, 15 individual sessions were excluded (due to transgressions of 11 subjects). In addition, one object-to-position retrieval test and one entire session 3 were excluded due to technical problems. Furthermore, eight people never performed the fifth session. All excluded or missing data were treated as "missing values."

Next, an outlier analysis was performed on the raw test scores. All scores that deviated from the mean by more then 3.29 standard deviations were replaced by a value of 3 standard deviations from the mean (19 replacements were made). After replacing the outliers, missing values were replaced by the group means for each variable.

\footnotetext{
${ }^{6}$ For session 2 , the exclusion criteria were: $<18 \mathrm{~h}$ or $>30 \mathrm{~h}$; for session 3 : $<5.25$ or $>8.75 \mathrm{~d}$; for session 4 : $<22.5$ or $>37.5 \mathrm{~d}$; and for session 5 : $<68.25$ or $>113.75 \mathrm{~d}$.
}

Memory performance is described in terms of hit rate, false alarm rate, $d^{\prime}$ score, and C (Snodgrass and Corwin 1988; Wickens $2002 a, b)$. Hit rate $(H)$ is the proportion of trained items that were correctly recognized (recognized items/trained items). False alarm rate (FA) is the proportion of foil items that were falsely recognized as list items (recognized foil items/presented foil items). $\mathrm{H}$ and FA were calculated for object memory, detailed object memory, object-to-position, and object-to-object memory. FA in response to new object foils and lures measured failures in general object recognition and detailed object recognition, respectively. In the case of object-position memory, "Yes" responses to objects from the training phase that had a different position during testing counted as false alarms. For object-to-object memory, "Yes" responses to recombined pairs counted as false alarms.

The $\mathrm{d}^{\prime}$ score is a discrimination measure, based on the Z-scores of $\mathrm{H}$ and FA. Since Z-scores of rates with a value of 0 or 1 are incalculable, response rates of 0 and 1 were converted to $1 /(2 \mathrm{~N})$ and $1-1 /(2 \mathrm{~N})$, respectively, where $\mathrm{N}$ is the number of trials on which the proportion was based (Macmillan and Creelman 2005). Then, $d^{\prime}$ was calculated as: $Z(H)-Z(F A)$. The larger $d^{\prime}$, the better a person is at discriminating between old and new items; $\mathrm{d}^{\prime}$ values near zero indicate chance performance. A negative $\mathrm{d}^{\prime}$ indicates that FA is higher than $\mathrm{H}$.

Response bias, indicated by $\mathrm{C}$, also incorporates $\mathrm{H}$ and $\mathrm{FA}$ and is calculated as: $-0.5[\mathrm{Z}(\mathrm{FA})+\mathrm{Z}(\mathrm{H})]$ (response rates of 0 or 1 were replaced as indicated above). When there is no response bias, $\mathrm{C}$ is 0 . A positive value indicates a conservative response bias (tendency to answer "No"); a negative value, a liberal response bias (tendency to answer "Yes").

The data were normally distributed and was analyzed through four separate repeated measures ANOVAs, one for each memory measure ( $\mathrm{H}, \mathrm{FA}, \mathrm{d}^{\prime}$, and $\left.\mathrm{C}\right)$. Each ANOVA incorporated two within-subject factors: "time" with five levels (the five retrieval sessions) and "memory component" with four levels (general object recognition, detailed object recognition, object-position associations, and object-object associations), or three levels in the case of $\mathrm{H}$ (general and detailed object recognition have the same H). Depending on a significant interaction effect between Memory component and Time, contrasts were specified to determine statistical differences between the decay of specific memory components. In these contrasts, the levels of the factor Memory component were contrasted against each other, while Time was represented as the first order (linear) polynomial, with the different retention intervals represented as $0.01,1,7,30$, and 91 days. When two memory components did show overall differential decay, a second type of contrast analysis was performed to assess differential decay over subsequent time steps in the retention period. In these analyses, each level in the factor Time was compared to the previous one (stepwise contrast).

\section{Acknowledgments}

This study was supported by grants 051-04-102 and 051-04-100 from the Dutch National Science Foundation (NWO). We thank Ralf Cornelissen for his assistance on web-based IT aspects of the project. We also thank Siebren van der Werf for his input regarding mathematical aspects of our results.

\section{References}

Aggleton JP, Vann SD, Denby C, Dix S, Mayes AR, Roberts N, Yonelinas AP. 2005. Sparing of the familiarity component of recognition memory in a patient with hippocampal pathology. Neuropsychologia 43: 1810-1823.

Alvarez R, Squire LR. 1994. Memory consolidation and the medial temporal lobe: A simple network model. Proc Natl Acad Sci 91: 7041-7045.

Anderson MJ, Riccio DC. 2005. Ontogenetic forgetting of stimulus attributes. Learn Behav 33: 444-453.

Bachevalier J, Nemanic S. 2008. Memory for spatial location and object-place associations are differently processed by the hippocampal formation, parahippocampal areas TH/TF and perirhinal cortex. Hippocampus 18: 64-80.

Battaglia FP, Pennartz CM. 2011. The construction of semantic memory: Grammar-based representations learned from relational episodic 
information. Front Comput Neurosci 5: 36. doi: 10.3389/ fncom.2011.00036.

Brown AS. 2002. Consolidation theory and retrograde amnesia in humans. Psychon Bull Rev 9: 403-425.

Catal LL, Fitzgerald JM. 2004. Autobiographical memory in two older adults over a twenty-year retention interval. Mem Cognit 32: 311-323.

Ebbinghaus H. 1885. Über das gedächtnis. Duncker \& Humblot, Leipzig, Germany.

Eichenbaum H. 2000. A cortical-hippocampal system for declarative memory. Nat Rev Neurosci 1: 41-50.

Hockley WE. 1991. Recognition memory for item and associative information: A comparison of forgetting rates. In Relating theory and data: Essays on human memory in honor of Bennet B Murdock (ed. WE Hockley, S Lewandowsky), pp. 227-248. Erlbaum, Hillsdale, NJ.

Hockley WE. 1992. Item versus associative information: Further comparisons of forgetting rates. J Exp Psychol Learn Mem Cogn 18: $1321-1330$.

Hockley WE, Consoli A. 1999. Familiarity and recollection in item and associative recognition. Mem Cognit 27: 657-664.

Kim JJ, Fanselow MS. 1992. Modality-specific retrograde amnesia for fear. Science 256: 675-677.

Lisman JE, Talamini LM, Raffone A. 2005. Recall of memory sequences by interaction of the dentate and CA3: A revised model of the phase precession. Neural Netw 18: 1191-1201.

Macmillan NA, Creelman CD. 2005. Detection theory: A user's guide. Lawrence Erlbaum Associates, Inc., Mahwah, NJ.

Manns JR, Hopkins RO, Squire LR. 2003. Semantic memory and the human hippocampus. Neuron 38: 127-133.

McAllister WR, McAllister DE. 1963. Increase over time in the stimulus generalization of acquired fear. J Exp Psychol 65: 576-582.

McClelland JL, McNaughton BL, O'Reilly RC. 1995. Why there are complementary learning systems in the hippocampus and neocortex: Insights from the successes and failures of connectionist models of learning and memory. Psychol Rev 102: 419-457.

Meeter M, Murre JMJ. 2004. Consolidation of long-term memory: Evidence and alternatives. Psychol Bull 130: 843-857.

Meeter M, Murre JMJ. 2005. TraceLink: A connectionist model of consolidation and amnesia. Cogn Neuropsychol 22: 559-587.

Meeter M, Murre JMJ, Talamini LM. 2004. Mode shifting between storage and recall based on novelty detection in oscillating hippocampal circuits. Hippocampus 14: 722-741.

Moscovitch M, Nadel L. 1998. Consolidation and the hippocampal complex revisited: In defense of the multiple-trace model. Curr Opin Neurobiol 8: 297-300.

Murre JMJ, Meeter M, Chessa AG. 2006. Modeling amnesia: Connectionist and mathematical approaches. In Statistical and process models for cognitive neuroscience and aging (ed. MJ Wenger, C Schuster), pp. 119-162. Erlbaum, Mahwah, NJ.

Nieuwenhuis IL, Takashima A. 2011. The role of the ventromedial prefrontal cortex in memory consolidation. Behav Brain Res 218: 325-334.

Perkins CC Jr, Weyant RG. 1958. The interval between training and test trials as a determiner of the slope of generalization gradients. J Comp Physiol Psychol 51: 596-600.

Peyrache A, Khamassi M, Benchenane K, Wiener SI, Battaglia FP. 2009. Replay of rule-learning related neural patterns in the prefrontal cortex during sleep. Nat Neurosci 12: 919-926.

Ribot T. 1881. Les maladies de la memoire. Germer Baillare, Paris.

Riccio DC, Joynes RL. 2007. Forgetting of stimulus attributes: Some implications for hippocampal models of memory. Learn Mem 14: 430-432.

Scoville WB, Milner B. 1957. Loss of recent memories after bilateral hippocampal lesions. J Neurol Neurosurg Psychiatry 20: $11-21$.
Snodgrass JG, Corwin J. 1988. Pragmatics of measuring recognition memory: Applications to dementia and amnesia. J Exp Psychol Gen 117: $34-50$.

Squire LR, Slater PC, Chace PM. 1975. Retrograde amnesia: Temporal gradient in very long-term memory following electroconvulsive therapy. Science 187: 77-79.

Squire LR, Clark RE, Knowlton BJ. 2001. Retrograde amnesia. Hippocampus 11: $50-55$.

Takashima A, Petersson KM, Rutters F, Tendolkar I, Jensen O, Zwarts MJ, McNaughton BL, Fernandez G. 2006. Declarative memory consolidation in humans: A prospective functional magnetic resonance imaging study. Proc Natl Acad Sci 103: 756-761.

Takashima A, Nieuwenhuis IL, Jensen O, Talamini LM, Rijpkema M, Fernandez G. 2009. Shift from hippocampal to neocortical centered retrieval network with consolidation. J Neurosci 29: 10087-10093.

Talamini LM, Meeter M. 2009. Dominance of objects over context in a mediotemporal lobe model of schizophrenia. Plos One 4: e6505. doi: 10.1371/journal.pone.0006505.

Talamini LM, Meeter M, Murre JMJ, Elvevåg B, Goldberg TE. 2005. Reduced parahippocampal connectivity produces schizophrenia-like memory deficits in simulated neural circuits. Arch Gen Psychiatry 62: 485-493.

Tse D, Takeuchi T, Kakeyama M, Kajii Y, Okuno H, Tohyama C, Bito H, Morris RG. 2011. Schema-dependent gene activation and memory encoding in neocortex. Science 333: 891-895.

Tunney JT. 2010. Do changes in the subjective experience of recognition over time suggest independent processes? Br J Math Stat Psychol 63: 43 62.

Vann SD, Tsivilis D, Denby CE, Quamme JR, Yonelinas AP, Aggleton JP, Montaldi D, Mayes AR. 2009. Impaired recollection but spared familiarity in patients with extended hippocampal system damage revealed by 3 convergent methods. Proc Natl Acad Sci 106: 5442-5447.

Wais PE, Mickes L, Wixted JT. 2008. Remember/know judgments probe degrees of recollection. J Cogn Neurosci 20: 400-405.

Wickens TD. 2002a. The equal-variance Gaussian model. In Elementary signal detection theory, pp. 17-38. Oxford University Press, Oxford.

Wickens TD. 2002b. The signal-detection model. In Elementary signal detection theory, pp. 3-16. Oxford University Press, Oxford.

Wiltgen BJ, Silva AJ. 2007. Memory for context becomes less specific with time. Learn Mem 14: 313-317.

Wiltgen BJ, Zhou M, Cai Y, Balaji J, Karlsson MG, Parivash SN, Li W, Silva AJ. 2010. The hippocampus plays a selective role in the retrieval of detailed contextual memories. Curr Biol 20: 1336-1344.

Winocur G, Frankland PW, Sekeres M, Fogel S, Moscovitch M. 2009. Changes in context-specificity during memory reconsolidation: Selective effects of hippocampal lesions. Learn Mem 16: 722-729.

Winocur G, Moscovitch M, Rosenbaum RS, Sekeres M. 2010. An investigation of the effects of hippocampal lesions in rats on pre- and postoperatively acquired spatial memory in a complex environment. Hippocampus 20: $1350-1365$.

Wixted JT. 2004a. On common ground: Jost's (1897) law of forgetting and Ribot's (1881) law of retrograde amnesia. Psychol Rev 111: 864-879.

Wixted JT. 2004b. The psychology and neuroscience of forgetting. Annu Rev Psychol 55: 235-269.

Yonelinas AP. 2002. The nature of recollection and familiarity: A review of 30 years of research. J Mem Lang 46: 441-517.

Zhou Y, Riccio DC. 1996. Manipulation of components of context: The context shift effect and forgetting of stimulus attributes. Learn Motiv 27: 400-407.

Zhu XO, Brown MW, McCabe BJ, Aggleton JP. 1995. Effects of the novelty or familiarity of visual stimuli on the expression of the immediate early gene c-fos in rat brain. Neuroscience 69: 821-829.

Received September 22, 2011; accepted in revised form March 28, 2012. 


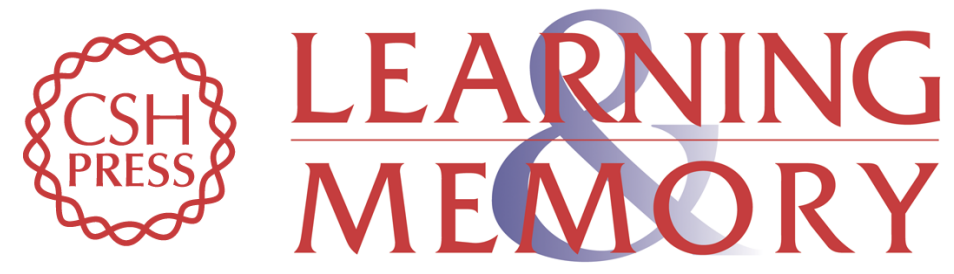

\section{Aging memories: Differential decay of episodic memory components}

Lucia M. Talamini and Eva Gorree

Learn. Mem. 2012, 19:

Access the most recent version at doi:10.1101//m.024281.111

Supplemental
Material http://learnmem.cshlp.org/content/suppl/2012/05/17/19.6.239.DC1

References This article cites 44 articles, 11 of which can be accessed free at: http://learnmem.cshlp.org/content/19/6/239.full.html\#ref-list-1

License

Email Alerting Receive free email alerts when new articles cite this article - sign up in the box at the Service top right corner of the article or click here. 